\title{
Historia de las mujeres en el Centro Médico ABC
}

\section{Woman history at the ABC Medical Center}

\author{
Paulina Seguí Vizcaíno*
}

\begin{abstract}
RESUMEN
La historia del Centro Médico ABC y de algunas de sus especialidades ha sido contada con anterioridad, pero nunca desde la perspectiva de la mujer. En el presente artículo se hace una referencia a la presencia de las mujeres desde su formación a principios del siglo XX y durante toda la evolución del hoy Centro Médico ABC. El objetivo de contar esta historia es tenerla por escrito y así futuras generaciones conozcan una historia hasta ahora no narrada. Es una recopilación de datos prácticamente de forma oral por diferentes departamentos del hospital, para comprender el papel de la mujer hoy dentro de la institución y, por lo tanto, su responsabilidad. Se describe la formación médica desde el internado rotatorio, el internado de pregrado y las residencias médicas desde sus inicios, así como la evolución de la Enfermería, las Damas Voluntarias, el Patronato y todos los departamentos donde las mujeres también están presentes, quienes incluso superan en número a los hombres en la actualidad. Conocer una misma historia desde otra visión nos acerca más a la nueva realidad que vivimos, y nos ayuda a comprender el papel que siempre ha desempeñado la mujer en diferentes departamentos dentro de la institución.
\end{abstract}

Palabras clave: Historia mujer, mujeres en el Centro Médico $\mathrm{ABC}$, mujeres en medicina.

Nivel de evidencia: $\mathrm{V}$

La Organización de las Naciones Unidas (ONU), ${ }^{1}$ en 1975, decidió conmemorar la lucha de la mujer por su participación en la igualdad de oportunidades en la sociedad y en su desarrollo integral como persona;

${ }^{*}$ Anestesióloga del Centro Médico ABC.

Recibido para publicación: 05/09/2019. Aceptado: 02/03/2020.

Correspondencia: Dra. Paulina Seguí Vizcaíno

Av. Carlos Graef Fernández Núm. 154-314, Col. Tlaxala,

05300, Alcaldía Cuajimalpa, CDMX.

Tel: 16647124

E-mail: pauseg2@yahoo.com.mx

\begin{abstract}
The history of de $A B C$ Medical Center and some of the medical specialities has been written before but this is the first time it is been told from a female perspective. The article is intended to show a different vision since its beginning as a hospital in the 1900s through our days being a Medical Center. The purpose of telling this story is that it stays written so future generations know a history not told till now. It is practically an oral recompilation department by department compiling information so we can know the women's role and her responsibility in the institution. It describes the history of the organization of nurses, doctors, Pink Ladies, Patronage throughout de evolution of the institution where women have play an important role and in this days there are even more women in some departments than men. Recognize a history from other perspective brings us closer to a new reality that we live today and help us understand the role that always has have women in different departments in all the $A B C$ Medical Center.
\end{abstract}

Keywords: Women history, ABC Medical Center history, medicine women.

Level of evidence: $V$

por eso, el 8 de marzo, en diferentes partes del planeta se celebra con pláticas sobre los logros alcanzados y las necesidades que todavía tenemos, ante este panorama, el Centro Médico ABC no fue la excepción. En el simposio Temas Actuales desde la Perspectiva de la Mujer se abordaron diferentes cuestiones que encontramos importantes de analizar desde otro ámbito que no fuera el médico y psiquiátrico, sino del medio ambiente, la psicología, la filosofía y la espiritualidad.

La historia de las mujeres en el Centro Médico $\mathrm{ABC}$ surge al percatarme de que pocas personas conocían la historia del hospital ${ }^{2} \mathrm{y}$, sobre todo, esta versión, y que como en cualquier crónica, un mismo acto puede analizarse desde diferentes perspectivas, 
generando nuevas ideas que pueden llevar a cambios positivos de la realidad.

El cuento es un género de la literatura que nos invita a imaginar, y dicen los que saben de esto que se apodera de nuestro inconsciente y se apropia de él, modificando nuestra realidad $;^{3}$ así que los invito a leer estas líneas en voz alta, como si le estuvieran contando un cuento a algún niño.

Como somos mexicanos, imaginemos el México de 1821, ya no pertenecemos a España, somos libres y en esta libertad que ahora intentamos desarrollar como personas y como nación, resulta que NO cualquier hombre puede ir a la universidad, ${ }^{4}$ sólo asisten los ricos, los influyentes, ningún indígena y pocos mestizos, a pesar de que en 1088 se abre la primera Universidad en Bolonia y en México en 1551, pocos hombres tenían el privilegio de recibir esta preparación. Pasan decenas de años para que cualquier mexicano pueda asistir a la universidad. Es en 1887 cuando Matilde Petra Montoya se recibe en medicina en la Real y Pontificia Universidad de México. ${ }^{5}$

La historia del Centro Médico ABC comienza con una mujer: Annie Cass (1860-1932), sufragista y filántropa que llega a México acompañando a su esposo: Weetman Dickinson, constructor inglés que viene a incursionar en el negocio del petróleo. En 1911, los Lords Cowdray deciden donar 100,000 libras para la construcción de un sanatorio y se funda el Sanatorio Lady Cowdray Home, al que la gente comenzó a llamar «Hospital inglés». Annie Cass convence a mujeres intrépidas a cruzar el Atlántico y venir a ejercer la enfermería, dejar su familia y sus costumbres para cuidar, curar, alimentar y hacer visitas a domicilio, así, en 1923 se funda el Sanatorio Cowdray con 14 camas.

En 1941 se une al Hospital Americano, fundado en 1886, curiosamente no hay mayores datos de esta creación, y nace como institución de asistencia privada The $A B C$ Hospital. Es importante mencionar que a casi 80 años de este suceso se le sigue conociendo como «Hospital inglés» y no como Centro Médico ABC, a pesar de ser un centro de especialidades desde $1997 ;{ }^{6}$ hago este paréntesis para hacer consciencia de cómo lo aprendido oralmente puede tener tanta influencia en la vida cotidiana del ser humano, de ahí la importancia del cuento oral.

Pero regresemos a nuestro cuento, en 1923 hay mujeres que cuidan enfermos, que no van a la universidad, pero que trabajan con habilidad, intuición y vocación para el cuidado de otras personas. Y en el hospital no sólo había enfermeras, sino que también trabajaban cocineras, las que se ocupaban de la limpieza; secretarias, es decir, las que ejecutan, no las que dan las órdenes, sino las que realizan el trabajo, a las que les gusta estar en contacto con las personas, cuidarlas y brindarles atención, aunque no hayan asistido a la universidad.

Ya son los años 30, nos liberamos del corsé, de las ataduras, usamos vestidos más cortos, nos mostramos más como realmente somos, pero los hombres también van más ligeros, ya no usan sombrero, ni guantes, ni chaqueta. $\mathrm{Y}$ a todos, hombres y mujeres por igual, nos van interesando otras cosas, vamos teniendo más inquietudes y, por lo tanto, queremos seguir aprendiendo. Algunas mujeres deciden tomar la LUCHA y convertirse en cuasi-hombres para poder ser escuchadas, tomadas en cuenta; mientras que otras continúan haciendo lo que ya llevaban años haciendo; así, en el ABC, se funda la Escuela de Enfermería en 1952, donde se formaliza la educación para el cuidado del enfermo y se otorga un título para poder ejercer la profesión. La Asociación Médica también se funda ese año, lo que significa que nos empezamos a organizar, y aunque entonces todavía no hay mujeres doctoras, pronto llegarán. Otras deciden ordenarse en su voluntariado y hacerlo formal y en 1953 nacen las Pink Ladies, increíble reunión de mujeres comprometidas, ejecutoras, que consiguen donaciones económicas, manejan una tienda donde todos los recursos son donados a diferentes programas dentro del hospital, dan su tiempo y en su historia sólo hay dos hombres. Y así ya llevamos alrededor de 65 años, tiempo durante el cual las mujeres han sido protagónicas, sin ellas este centro médico no existiría y por supuesto de la mano, a la par, en igualdad de decisiones que los hombres, que los médicos, que los patronos, que los directores.

Ya llevamos más de 100 años de libertad y estamos más organizados como sociedad, se descubre el $\mathrm{ADN}$, la televisión a color, y debido a las guerras las mujeres tuvieron que salir a trabajar, por lo que comienzan a ser más las que asisten a más universidades y que tienen más oportunidades; pero todavía hay mucha desigualdad en salarios, condiciones de trabajo, etcétera.

Más mujeres comienzan a estudiar medicina y hacer especialidad. En 1942 inician las residencias en México en los hospitales de salubridad ${ }^{7}$ y unos años después el $\mathrm{ABC}$ se une a la oferta. Quienes llevan más de 50 años aquí, recuerdan a dos mujeres: la Dra. Holden, pediatra y la Dra. Talamás, cirujana plástica.

La Dra. Melman, anestesióloga pediatra, ingresa en 1968 y la Dra. Gerson, oncóloga, hasta 1980. Pasan 12 años sin ninguna otra doctora, la razón: 


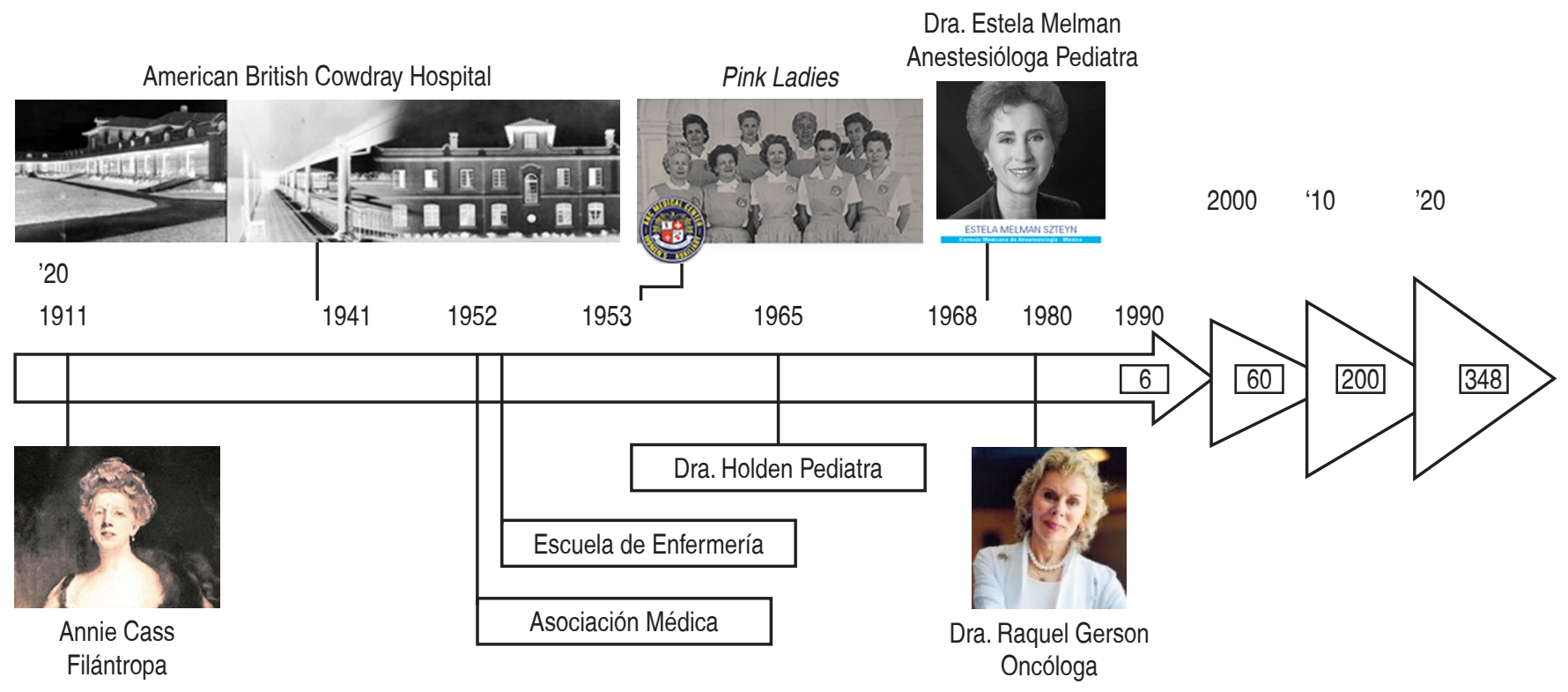

Figura 1: Línea del tiempo en la historia de las mujeres en el Centro Médico ABC, desde su fundadora hasta nuestros días.

ninguna cumplía con los requisitos para ingresar al cuerpo médico o, simplemente, no les interesaba la medicina privada. Y después cada dos años se aceptan una, dos, tres mujeres; desde 1994 cada año son aceptadas más; para 2010 éramos 200 y desde entonces han aceptado a otras tantas (Figura 1). Actualmente, de 1,400 médicos del personal somos $392 \mathrm{mu}-$ jeres, lo que representa $28 \%$ y de éstas, 55 tienen una subespecialidad, es decir, $14.03 \%$.

Ahora bien, regresemos un poco en el tiempo a las residencias médicas, el hospital innovador y siempre evolucionando abre las primeras residencias, y cuál fue mi sorpresa al encontrar:

1976 se abre Cuidados Críticos con una única mujer. 1986 Medicina Interna: una mujer y dos hombres. 1986 Ginecología: segundo año, una mujer.

1986 Cirugía: tercer año, dos mujeres.

1986 Patología Clínica: quinto año, una mujer. 1989 Anestesia: una mujer y un hombre.

1991 Radiología: tercer año, dos mujeres.

1991 Ortopedia: 12 años después, primera mujer.

En este siglo se han abierto subespecialidades en las que están integradas las mujeres, existen 16 especialidades y 13 postgrados. Que no haya hoy en día en alguna generación es porque no llegaron, no porque se les niegue la plaza por ser mujeres. Que existan residencias donde las mujeres no queramos ejercer, me queda más claro hoy que nunca. No exis- ten mujeres urólogas de hombres en el hospital, pero sí de subespecialidad de ginecología, y sí, todas, TODAS hemos tenido que estudiar, trabajar, demostrar nuestros conocimientos y habilidades, IGUAL que TODOS los hombres.

Entre los internos de los rotatorios de pregrado como actualmente es y los residentes rotatorios de los años 70 tenemos paulatinamente mayor participación de las mujeres, pero desde el inicio el hospital dio oportunidad a las mujeres de prepararse aquí (Figura 2).

Como hemos visto, las enfermeras ocupan un papel importante en la historia del hospital, aquí

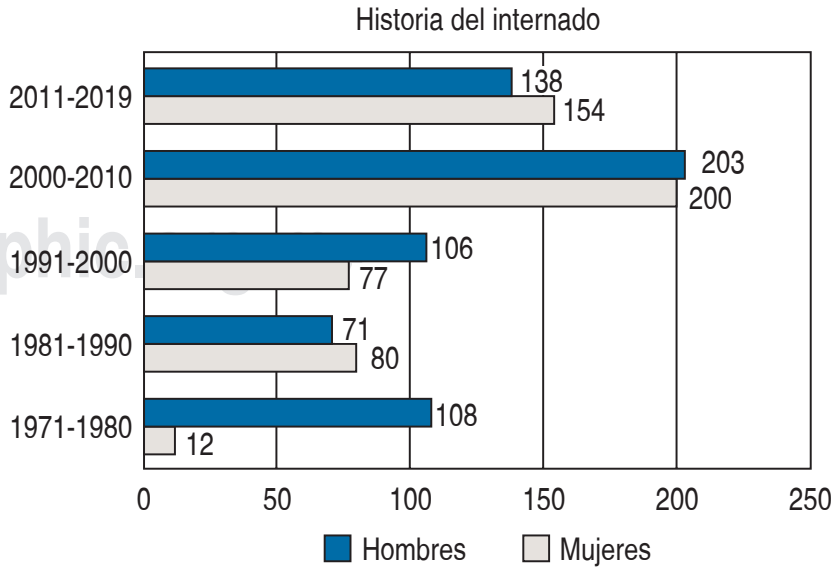

Figura 2: Clasificación según género de internos rotatorios y de pregrado que terminaron su rotación en el Hospital/Centro Médico. 
Historia del Patronato

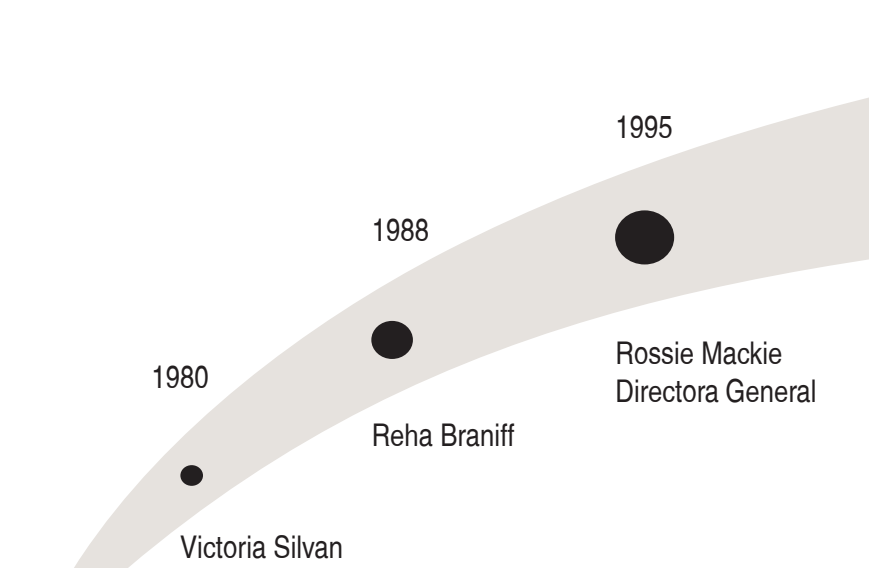

la equidad de género está en clara desventaja con los hombres. Por cultura, como me dicen las jefas, no pueden poner a ningún hombre en el hospital de pediatría y ginecología. Y continuamos como hace 50 años, que es más o menos lo que recuerdan las enfermeras de mayor antigüedad en el hospital, los pocos hombres que hay están en los lugares donde los pacientes no pueden verlos o, más bien, opinar al respecto: quirófano, Unidad de Terapia Intensiva y urgencias. Esto es un punto muy importante para evolucionar en este micromundo del $\mathrm{ABC}$, que es un reflejo de lo que sucede afuera, los hombres también merecen las mismas oportunidades y su género no debe ser una razón por la que todos los pacientes son atendidos por hombres doctores.

El patronato, los que trabajan por vocación de servicio para que al paciente, a los médicos, a las enfermeras y a todos los que laboramos aquí nos siga yendo bien y por quien perduramos con los principios originales de aquel «Hospital inglés», también cuenta ya con mujeres (Figura 3).

Y la lista continúa, hoy existen mujeres en todos los servicios del hospital, desde los años 90 fuimos ocupando más espacios, más gerencias, más jefaturas, estamos en todas partes, con carreras técnicas y universitarias (Tabla 1).

Y por esta razón, me gustaría terminar con una metáfora, otro método educativo ancestral retomado por Erickson, ${ }^{8}$ que se utiliza también en los cuentos infantiles y en las enseñanzas espirituales. El ahuehuete es el árbol nacional de México, viene del náhuatl hue: viejo, huehue: grande y atl: agua y los invito a que consideremos lo siguiente:
Figura 3:

Historia de las mujeres dentro del Patronato del Centro Médico ABC.

Las raíces del árbol son esas 14 mujeres que, junto con Annie Cass y todos los hombres que creyeron en el proyecto, nos anteceden y nos sostienen firmemente en la tierra. El tronco es todo lo que sucedió el siglo pasado, los hombres y las mujeres que fueron a preparase, que se organizaron y que nos sostienen en los valores y principios de la institución, y las ramas con sus zhojas somos nosotros, las mujeres y los hombres que continuamos preparándonos, aprendiendo y evolucionando para seguir sanando, curando, alimentando a los enfermos en cuerpo y alma.

Tabla 1: Cargos ocupados por mujeres en febrero de 2019 representan $52.41 \%$ de la planilla total de trabajadores.

\begin{tabular}{lc}
\hline Cargo & Número de mujeres \\
\hline Administrativos & 263 \\
Servicios alimenticios & 97 \\
Médicos & 93 \\
Puestos de coordinación & 65 \\
Servicios de limpieza, suministros & 64 \\
Química farmacéutica & 54 \\
Jefaturas & 54 \\
Supervisoras & 53 \\
Laboratoristas & 40 \\
Odontología, nutrición, trabajo & 39 \\
social & \\
Rx e imagen & 37 \\
Terapia física & 34 \\
Gerencias & 26 \\
Hemodinamia & 22 \\
Biólogas, investigadoras & 8 \\
\hline
\end{tabular}


Seamos conscientes de que HOY las mujeres SÍ ocupamos, practicamos y trabajamos en donde queremos.

Seamos conscientes de impulsar el crecimiento y aprendizaje de las niñas en todas las ciencias y en todas las profesiones en igualdad de circunstancias.

Seamos conscientes de MEJORAR nuestros lugares de trabajo con respeto y educación.

Deshacernos de creencias e historias de siglos pasados es la clave para mejorar como persona y, por lo tanto, como sociedad.

Reaprender lo aprendido desde otra perspectiva es evolucionar.

Responsabilizarnos por nuestro entorno, tener la valentía de denunciar, dejar de ser víctima y convertirse en persona responsable de sus actos es también lo que hará que el sistema de desigualdad que hoy persiste termine.

$\mathrm{Mi}$ agradecimiento infinito en la realización de este trabajo a todas las personas que entrevisté, hombres y mujeres con memoria extraordinaria para ordenar el cuento, y agradezco especialmente a:

- Rosario Castro Trejo del Departamento de Enseñanza, quien ha guardado, ordenado, clasificado todos y cada uno de los documentos, libretas y libros consultados para poder sacar las estadísticas de todos los médicos que nos hemos formado en la institución durante 30 años.
- Beti Castelán (gerente de asuntos médicos), que ha hecho una labor titánica al ordenar, guardar y acompañar a todos los médicos; sin su memoria de la historia este escrito no podría existir.

- Blanca de la dirección de enfermería, que contactó con enfermeras retiradas reuniendo más historias de mayor tiempo atrás.

- A ti lectora/lector, por dedicar un tiempo a escuchar un cuento y darte la oportunidad de reaprender para mejorar.

\section{BIBLIOGRAFÍA}

1. Naciones Unidas. Día Internacional de la Mujer, 8 de marzo. Web de Naciones Unidas. 2019.

2. Fernández-Vázquez JM. Historia del Centro Médico ABC. An Med Asoc Med Hosp ABC. 2012; 57 (1): 68-78.

3. Sarabia-García FA. El cuento como herramienta psicoterapéutica en el manejo emocional de niños con discapacidad. Rev Electrónica Psicol Iztacala. 2012; 15 (4): 1209-1223.

4. Marsiske R. Historia de la Universidad de México: historia y desarrollo. Rev Hist Educ Latinoam. 2006; 8: 9-34.

5. Rodríguez de Romo AC, Castañeda-López G. Inicio de las mujeres en la medicina mexicana. Rev Fac Med (Méx.). 2015; 58 (2): 36-40.

6. Fernández-Vázquez JM, Camacho-Galindo J, Álvarez-Salas FS. La ortopedia y traumatología en el Centro Médico ABC: historia. An Med Asoc Med Hosp ABC. 2017; 62 (1): 74-79.

7. Quijano-Pitman F. Origen y desarrollo de las residencias hospitalarias. Gac Méd Méx. 1999; 135 (1): 73-76.

8. Cardona-Galeano I, Osorio-Sánchez Y. Uso de la metáfora en terapia familiar. Aportes al enfoque narrativo. Rev Virtual Univ Católica del Norte. 2015; 44: 15-35.

\section{Fe de Erratas}

En el artículo Resultados del diagnóstico temprano de sordera en el recién nacido con factores de riesgo en el Centro Médico $A B C$, publicado en el volumen 64, número 4 del 2019 (pp. 252-259), el nombre del último co-autor dice: Roberto Richheimer Watson; debe decir: Roberto Richheimer Wohlmuth, cuya especialidad es Pediatría. 